\title{
A Self-aligned Gate Definition Process with Submicron Gaps
}

\author{
L.F.P. Warmerdam, A.A.l. Aarnink, J. Holleman, H. Wallinga \\ University of Twente, IC Technology and Electronics Department, \\ P.O. Box 217, 7500 AE Enschede, Netherlands
}

\begin{abstract}
A self-aligned gate definition process is proposed. Spacings between adjacent gates of $0.5 \mu \mathrm{m}$ and smaller are fabricated. The spacing is realized by an edge-etch technique, combined with anisotropic plasma etching of the single poly-silicon layer. Straight gaps with minor width variation are labricated. Minority carrier life-time and breakdown voltage are not affected.
\end{abstract}

\section{Introduction}

In order to completely integrate complex CCD-based functions, a combined BCCD-CMOS process has been developed. The main research area for circuits fabricated in this process is video frequency filter applications. The low-voltage n-channel BCCD-CMOS process is fully ion-implanted and uses a self-aligned gate definition process. In contrast to an overlapping gate technology, this reduces the inter-electrode capacitances considerably and avoids electrical isolation problems with the dielectric between first and second poly-silicon layer. Furthermore all four phases are identical using this approach. Definition of the sub micron gaps is oblained by technological means, rather than by advanced optical lithography or eleclron-beam lithography [1]. In fact, the demands on the lithographic process are not determined by the dimensions of the gap but by the minimum feature size used.

A gap between adjacent gates leads to a local maximum in the potential of a depleted BCCD channel when both gate vollages are equal. This maximum functions as a well in which a fraction of the transported charge packets is trapped, causing an increase in the charge-transfer inefficiency. In figure 1 the simulated values of the well potential as a function of gap width is shown. The potential maximum is somewhat bias dependent and vanishes rapidly with increasing potential difference between adjacent gates. Due to this effect are small well potentials tolerable. Simulations have shown that well polentials up 
to $0.5 \mathrm{~V}$ originating from gap widths up $10.5 \mu \mathrm{m}$ do not seriously degrade charge transler performance.

\section{Processing}

For the tabrication $\langle 100\rangle$ p-type epitaxial walers were used. Standard LOCOS isolation is combined with high energy ion implantalion of both $p$ - and $n$-well. The gate definition process is based on the edge-etch technique [2,3]. Figure 2 shows the process flow of the gate definition. Gate oxide thickness is $25 \mathrm{~nm}$, poly-silicon thickness is $500 \mathrm{~nm}$. Key step is the isotropical back-etch of the silicon dioxide layer, particularly regarding the lateral etching of the silicon dioxide at the edges (step 4, fig. 2a). The amount of etching at this step determines the width of the elch-mask of the gap. Therefore the gap width can be monitored easily by adjusting the etch time; spacings in the deep sub nicron regime are leasible. The etch of the silicon dioxide and silicon nitride stack (step 3, GD mask) is performed anisotropically in a $\mathrm{CHF}_{3}-\mathrm{O}_{2}$ plasma. The lateral back-etch of the silicon dioxide layer (step 4) is perlormed in a thermostated high-purity $\mathrm{HF}-\mathrm{NH}_{4} \mathrm{~F}$ mixture and is completely isotropic. The lact that lateral elching is performed at a nearly perpendicular oxide side-wall results in homogeneous and reproducible etch behavior. This implementation yields straight gaps with minor width variation.

All features defined in slep 3 using the GD mask automatically result in poly-silicon patterns. Single poly-silicon leatures are defined in slep 7 by etching a pattern in the masking oxide. An overlap of PS and GD masks results in self-aligned poly-silicon gates. Separation of the gates is warranted by the gaps, which are present at the edges of every GD leature. The gates for BCCD devices and CMOS transistors are elched in a single step. The poly-silicon is etched anisotropically in a $\mathrm{Cl}_{2}-\mathrm{SiCl}_{4} \mathrm{RIE}$ plasma with high selectivily over silicon dioxide. The integrity of the gate oxide in the gap is restored by a thermal oxidation (fig. 2d). Simultaneously the side walls of the poly-silicon gates are oxidized. The gaps are filled with a planarizing oxide. Finally a slandard single-layer metal back-end process is used to complete water fabrication.

\section{Results}

The figures 3 and 4 show SEM pictures of $0.5 \mu \mathrm{m}$ gap devices on a wafer, extracted from processing directly after the gate definition process. Figure 3 presents a detail of a test device with gaps running mcander-like over the active area. Clearly the gaps run in straight lines and show minor width variation. Corners and lopographic steps at the bird's beak do not influence gap 
dimensions. Gaps always end at the edge of poly-silicon features. At those points a direct transition exists from sub-micron spacing to open area.

In figure 4 a cross-section of the gap is shown. A poly-silicon capping layer has been deposited for contrast enhancoment only. Due to the thermal oxidation treatment after the gap fabrication a firm passivaling oxide layer exists on side walls and exposed substrate. The silicon nilride between the silicon dioxide layer and poly-silicon gate (situated at the top right side of the gap, the silicon nitride layer is emphasized by means of a drawn line) prevents the complete encapsulation in silicon dioxide of the gate. This poses no problems concerning electrical isolation. In normal processing the gap is filled with an insulator.

Several wafers with test devices have been realized. Dovices had gap widihs of 0.2-0.5 $\mathrm{km}$. C-t measurements have been perlormed on capacitances with and without gaps meandering over the surface. For both type of devices minorily carrier lifetimes of $20 \mu \mathrm{s}$ have been found. Also no difference in breakdown voltage has been observed. Breakdown voltage is $23 \mathrm{~V}$. With a gate-oxide of $25 \mathrm{~nm}$ a breakdown field strength of $9.10^{6} \mathrm{Vcm}^{-1}$ is found. If is concluded that the damage caused by plasma elching is removed sulficiently.

\section{Acknowledgements}

This work has been supported by the Dutch Foundation for Fundamental Research on Matter, FOM. The authors would like to thank G.Boom and B.Otlen for their assistance with SEM photography and F.W.Ragay and R.C.M.Wijburg for numerous encouraging discussions.

\section{References}

1. J.W. Slotboom et. al.; "Sub micron CCD Memory structures labricaled by electron-beam lithography"; IEDM Tech.Dig. 1984; 308-311

2. H.H. Hosack and R.H. Dyck; "Sub micron Patterning of Surlaces"; IEEE Journal of Solid-State Circuits; SC-12(4) aug. 1977; 363-367

3- V.J. Kapoor; "Charge-Coupled Devices with Sub micron Gaps"; IEEE Electron Device Letters; EDL-2(4) april 1981; 92.94 


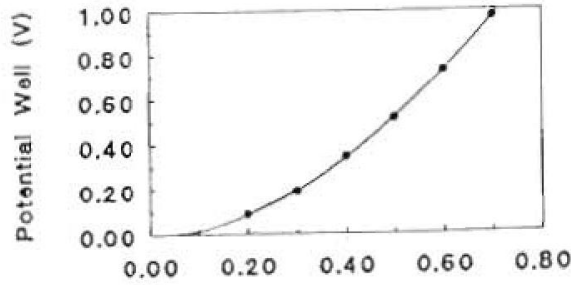

Gap Width (um)

Figure 1: Simulated values of the potential well below the gap as a function of the gap width. Gate voltage on both gates was ov.

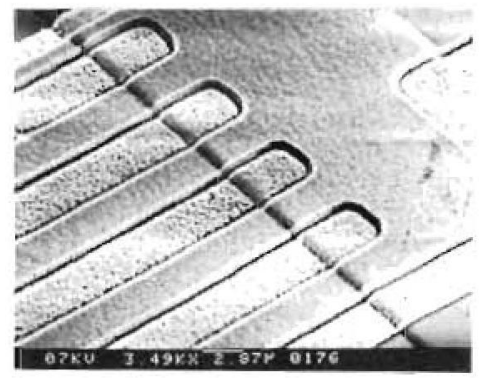

Figure 3: SEM photograph of a detail of a test device.

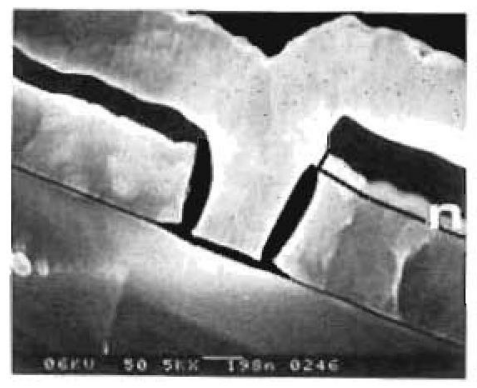

Figure 4: SEM photograph of a cross section of the gap after the gate definition process. A poly-silicon capping layer has been deposited. On top of the right hand gate, the presence of a silicon nitride layer is emphasized by the drawn line.

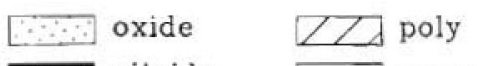

ERif nitride $\square$ mono

a
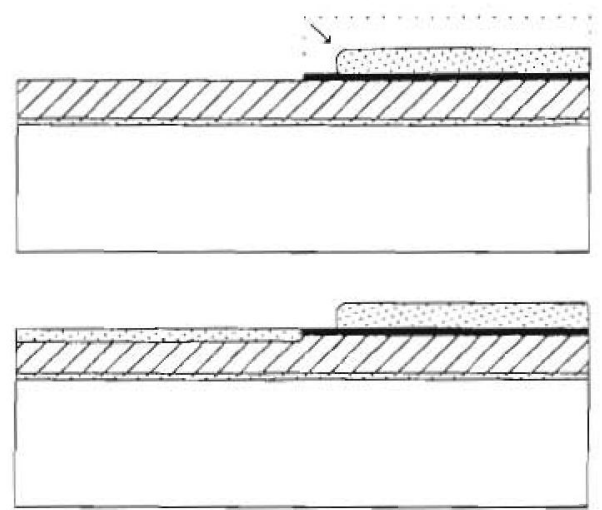

C
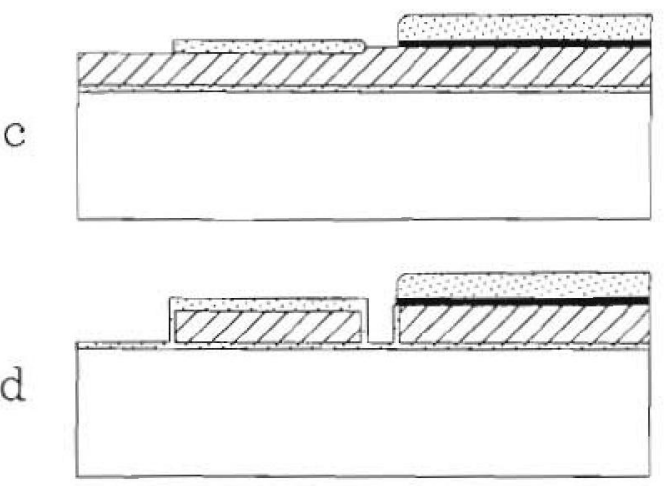

Figure 2: Process flow of the gate definition.

1. Starting point: LOCOS, implanted wells, $25 \mathrm{~nm}$ gate oxide

2. Deposition of poly-silicon, doping, deposition of silicon nitride and silicon dioxide

3. Definition of the silicon dioxide and silicon nitride stack, Gap Definition (GD) mask, resist strip

4. Back etch of sillicon dioxide (fig. a)

5. Growth of a thermal oxide on the exposed poly-silicon. (fig. b)

6. Etch of the exposed silicon nitride rims

7. Definition of the masking oxide, Poly-Silicon (PS) mask, resist strip (fig. c)

8. Etch of poly silicon

9. Growth of passivating oxide (fig. d) 